\title{
Implementation System Telegram Bot for Monitoring Linux Server
}

\author{
Mohammad Idhom ${ }^{1}$, Ronggo Alit, Henni Endah \\ Wahanani \\ Department of Informatic Engineering \\ Universitas Pembangunan Nasional "Veteran" Jawa Timur \\ Surabaya, Indonesia \\ 1idhom@upnjatim.ac.id
}

\author{
Akhmad Fauzi \\ Department of Management \\ Universitas Pembangunan Nasional "Veteran" Jawa Timur \\ Surabaya, Indonesia
}

\begin{abstract}
Security is an essential aspect of information, especially in a network that is connected to the internet. Threats from inside or outside often disrupt network security. Telegram is a cross-platform messaging system that centers on the security and privacy of security and privacy of the wearer. The problem that often occurs in a company or institution that has a server is the lack of flexibility in the supervision process due to issues with human error, especially the admin server that is in charge of supervising server performance so that it is less able to observe the server for $\mathbf{2 4}$ hours. Looking at these problems, it is necessary to have a system that can perform remote monitoring processes to increase admin server flexibility. The system can monitor the server and send notifications. Notifications sent as information contained on the server and integrated in the telegram application. The information provided comprises uptime, Central Processing Unit (CPU) usage, ram usage, swap usage, active users, disk usage, user's login and logout. The use of Monitoring integrates the Telegram Application Programming Interface (API) function into it to be able to send messages and check in realtime. With the establishment of a Monitoring System that is equipped with checking and monitoring to the network more optimally, because of the integration between systems that are directly connected to the System Administrator.
\end{abstract}

Keywords - security; telegram boot; monitoring introduction

\section{INTRODUCTION}

Smartphones appear as a practical tool for use in daily activities and large events including social interactions. This is because smartphones give users access to strong services and resources through small media [1]. This makes Messenger applications in smartphones appear as many important tools in communicating. Instant messaging is used because it is a means of communicating which is one of the main human activities. Do and Gatica-Perez also mentioned that social interaction is an important role in our daily lives as the largest part of our activities [2].

Network monitoring is a routine process of collecting data and measuring the progress of a network that monitors any changes that occur to maintain existing network management by knowing whether or not the devices are connected to the network [3].

The results of Hill research [4] are used as a benchmark for how language needs to be used in the chatbot design. As well as referenced some evaluations that need to be considered from the results of Hung research [5] in the analysis and design of new chatbots such as ease of usage, clarity, naturalness, friendliness, robustness regarding misunderstandings, and willingness to reuse the system

Most companies that are still developing have problems with the server, especially the issue of supervision, while servers are fatal if not monitored properly. Large companies that have a shift schedule in supervising servers sometimes experience negligence problems so they need a quick way to communicate with the supervising server. In this case, you need fast notification.

Therefore, to overcome the existing problems, it is necessary to build a "Mobile Monitoring System for Linux Environment Server by Using Telegram Bot" with the aim that the System can monitor the server and make it easier to check. Notifications sent in the form of messages as information and integrated in the telegram application that can be accessed via a smartphone. The system will be integrated between systems that are directly connected to the System Administrator.

\section{RELATED WORKS}

\section{A. Telegram Bot}

The Telegram application was chosen because this application is free, lightweight and multiplatform. Telegram also has a Bot API that is quite complete and growing, making it possible to create smart bots that can respond to messages from the public [5]. Bot Response is designed dynamically by utilizing the resources used in Smart Campus. All messages from students will be recorded and classified to be used to respond to subsequent messages

There are two ways to create a Telegram Bot, using the long polling and webhook methods. With the long-polling method, the server checks periodically to the bot if there is an incoming message. If there is a message coming in, the server will execute based on the request message sent by the user. If there is no message, the server condition is idle. In the LongPolling method Bot will run directly through the server, while 
in the Webhook bot method it will be in hosting and must use https [6].

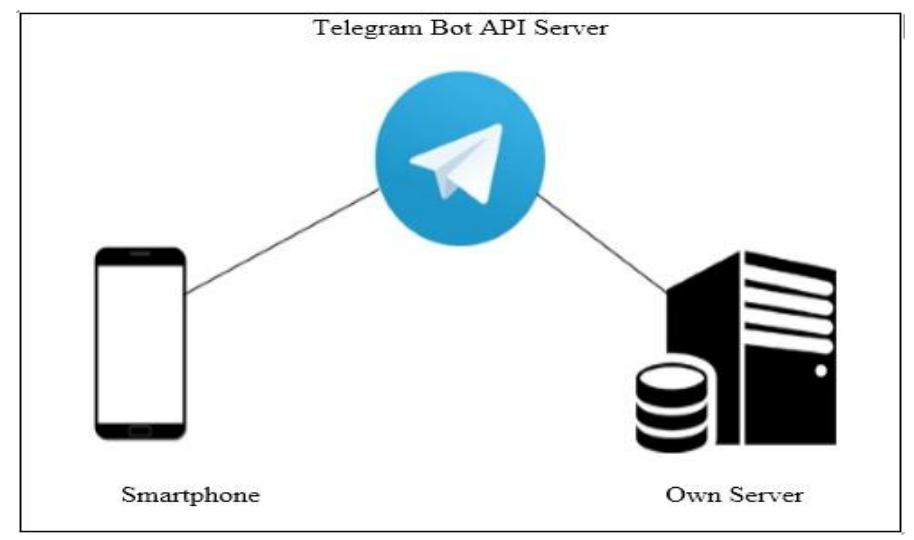

Fig. 1 Telegram Bot Architecture

Fig 1. Describes the smartphone communicating with the Telegram server as a bridge between the client and the server bot. There are two main terms used, namely requests and updates. The Admin server requests via the Telegram server as a bridge between the mobile device and the supervised server, then the Telegram server runs the polling process with the HTTPS protocol into the server and the bot server provides updates from the results of Admin Server requests. In order to do this, the design is done using the Telegram Bot API as an intermediate medium for Admin Server. Telegram Bot API is an API (Application Programming Interface) that is used to remotely monitor using bots as programs running on the server. Telegram Bot API uses the MTPproto encryption protocol that has been prepared for developers as a means of security.

\section{B.Linux}

Linux is an open source operating system and load (free) below GNU (GNU is Not Unix) GPL (General Public License) license. The meaning of open source is the source code (source code) included in the Linux program so that can be seen by anyone without having to sign a special agreement such as NDA (Non-Disclosure Agreement).

The meaning of the word free or free refers to freedom not to freedom cost. Linux is free to duplicate, modify, and distribute well for non-commercial or commercial purposes, and this is valid under the GNU license GPL to get Linux, of course, has to cost, but costs it is not to pay the license for using Linux, but for duplication, or shipping, or service fees, or a combination of all three. [7]

\section{Python}

The Python was created by Guido van Rossum in the Netherlands in 1990 and his name was taken from the television program Guido Monty Python's Flying Circus. Van Rossum developed Python as a hobby, then Python became a programming language that is widely used in industry and education because it is simple, concise, intuitive, and has a broad library of syntax [8].

\section{Github}

Github is a popular web service for software development projects that use Git version control systems or hosting services for open source projects that use the Git control revision tool system. Easy understanding, Github is a social network for developers in developing a project

This github site provides social networking and social networking graphics functionality to show how developers work on their repository versions. Github also operates other services: a pastebin-style site called Gist that presents wikis for each repository and web pages that can be edited through a Git repository, slide hosting service called Speaker Deck, and a web analytics platform called Gauges.

\section{E. VirtualBox}

System Software is software or software that deals directly with computer hardware components or hardware, maintenance, and programming. VirtualBox is a virtual machine application that allows you to install other Operating Systems, and run simultaneously on the parent operating system. VirtualBox is an open source application from Sun MicroSystem (now acquired by Oracle) that is targeted at servers, desktops and embedded uses. VirtualBox is a virtualization software, which can be used to execute additional operating systems in the main operating system. For example, if someone has an MS Windows operating system installed on his computer, then that person can also run another desired operating system in the MS Windows operating system.

\section{F. Ubuntu Server}

Ubuntu Server is an operating system derived from Ubuntu Linux specifically designed with a customized kernel to work as a server operating system. Linux Kernel Ubuntu Server is specifically designed to work with more than one process (multiprocessor) with NUMA support at $100 \mathrm{~Hz}$ internal timer frequency and using scheduling deadline I / O. Linux Ubuntu Server has an open source license and is free and is a derivative of the Debian Linux distro so that it has high security. Also, any bugs related to security are quickly handled by the Ubuntu Linux security team that works with the Debian security team. Linux Ubuntu Server has a minimum requirement or resources that must be met including a $300 \mathrm{MHz}$ processor, $64 \mathrm{MB}$ memory, $500 \mathrm{MB}$ hard drive and VGA $640 \times 480$. However, to run applications with large enough computing, it is better to have resources on the computer provided higher to improve performance on the application

\section{III.METHODOLOGY}

In this study using the PPDIOO method (Prepare, Plan, Design, Implement, Operate, Optimize.

In the design of this system, it is explained that the stages in making a monitoring system start from the installation of the operating system used on the PC server then the installation of the supporting application. In designing this system the server uses the Ubuntu Server operating system, After Ubuntu Server is installed on the server and telegram on the smartphone. As in Fig 2 below: 

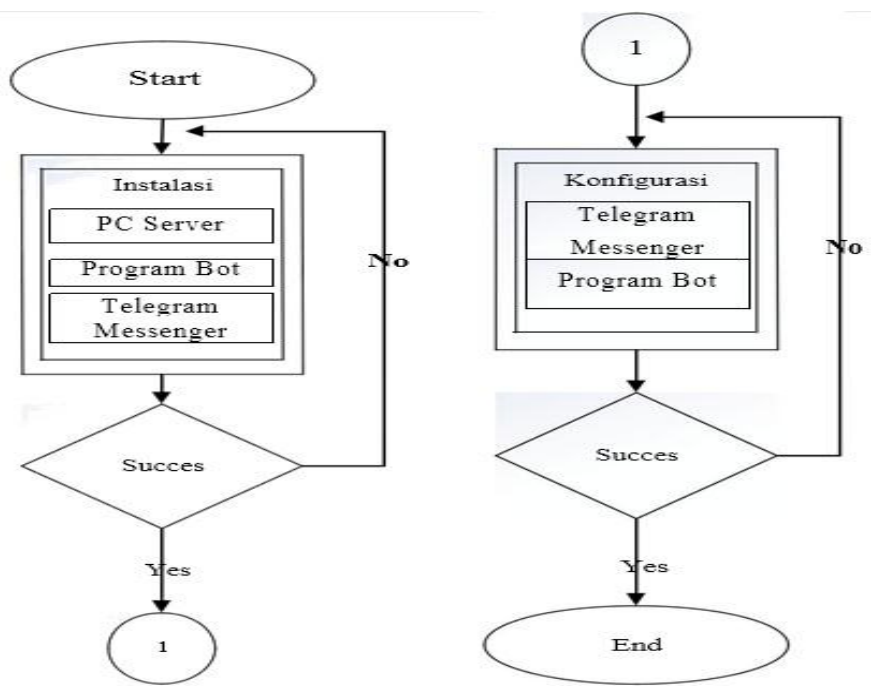

Fig. 2 System Test Flow

Bot is a program that runs on the side of the server and to get information by using the Telegram Client that is installed on the mobile admin server. The use of Telegram Client serves as an interface that displays certain information. The function of the bot can be seen in Fig 3 below:

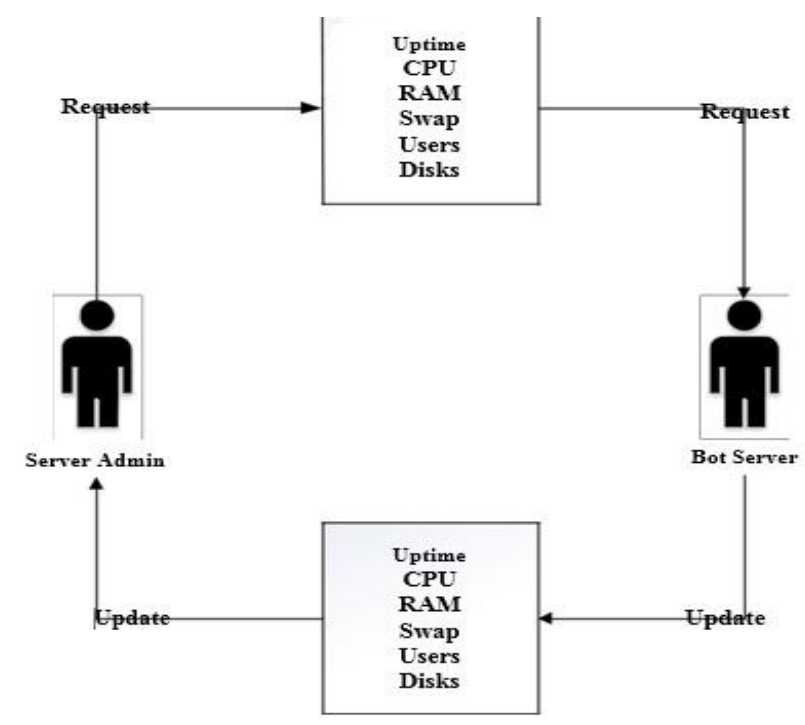

Fig. 3 System Trial Flow

Describes the role of the admin server and server bot. The server admin can request Uptime, CPU, RAM, Swap, Users and Disks. The server bot can send information that has been requested by the admin server and send notifications in the Uptime, CPU Usage, RAM Usage, Swap, Active Users and Disks Usage section.

In the process that is run, two processes work, namely the request and update. The request functions as a request from the admin server to get the information needed while the update process functions as a reply from the bot server as a result of the process that has been requested by the admin server. The system request workflow can be seen in Fig 4 below:

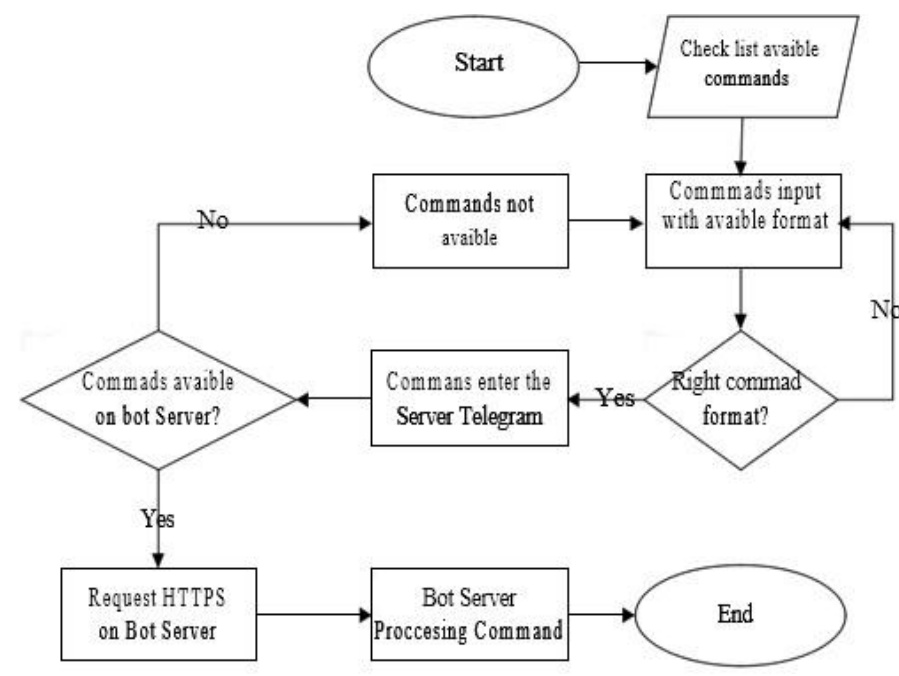

Fig. 4 Proses Request Telegram Bot API

The bot will check to enter the available command or not; then the command will go to Telegram Server as a link between admin and bot. Then the server bot telegram ensures that the command input is valid or the command format is correct, the command will go to HTTPS into the server and then the server bot receives a request command and performs the command process.

The request process has been completed and will return to the admin side, the server bot running on the server side will return to the server admin side through the update process. The update process will run in a few seconds when the admin requests. In the update process as in Fig 5 below

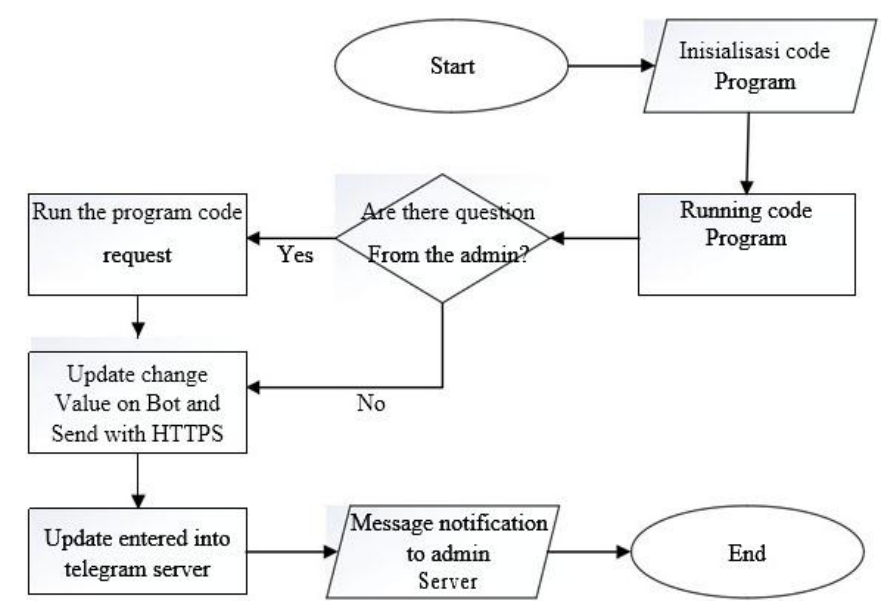

Fig. 5 Update Process in Telegram Bot API

The update process is the process of sending results from the request process. The update process begins with the process where the bot program runs on the server. Then the existing program code initializes the program. Then the bot will check the admin request. Then the bot does its job by observing the changes in values that occur inside the server and immediately 
sends the results to the server admin via the Telegram Server. After going through the Telegram server, the results will be sent to the Telegram client that the admin has in the form of a notification or message.

\section{RESULT AND DISCUSSION}

The bot will be able to recognize or accept several commands that can be used by users, including:

- /start - The first command that will be sent by the user if the user has never used a bot.

- /help - The command to display help contains the menus available on

- the bot. / uptime - This command will display the length of time after the server is turned on. / CPU Command to display the CPU used by the server.

- $\quad /$ ram - Command to display RAM used by the server.

- /swap - Commands to display Swap that the server uses.

- /users - Command to display active users along with login time. / disks - A command to display the disks that the server uses.

- $\quad$ /stop - Command to terminate the server monitoring system.

\section{Command /start}

The /start command will be used when the user first interacts with this bot then making this command is also important, if the command cannot be done properly, the bot will not function properly. As in the following Fig 6:

21:13
$\begin{aligned} & \text { Monitoring Linux } \\ & \text { bot }\end{aligned}$
Silahkan Masukkan Password yang
Benar. Ketik/start <password>
untuk Sign In.

Fig. 6 Command Start

\section{Command /help}

The /help command is used when the user gets further information. This information is in the form of a feature in the bot. As in Fig 7 below:

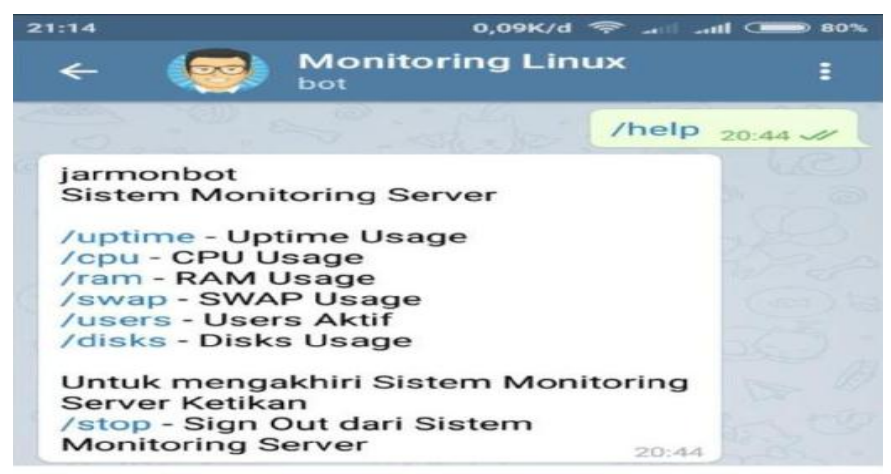

Fig. 7 Command /help

\section{Command /ram}

This command displays the RAM used on the server. Like on following Fig. 8:

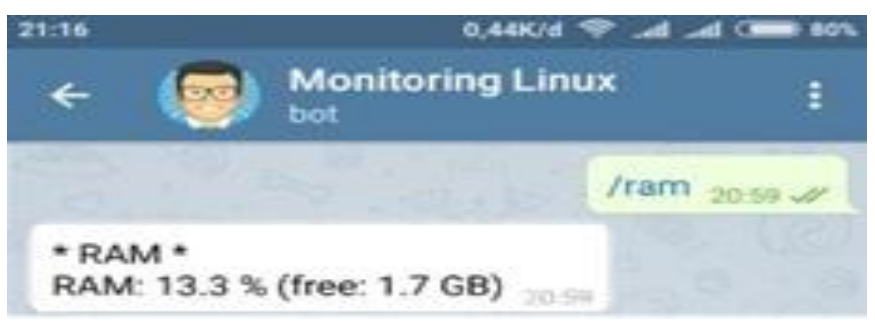

Fig. 8 Command /ram

RAM checking is also done manually; it aims to find out the notification goes according to the function that has been made or not. As in Fig 9 below:

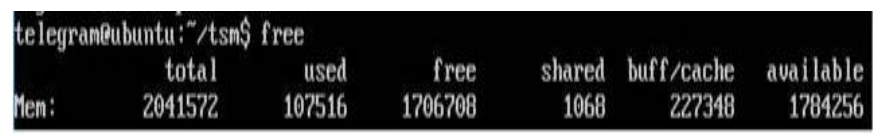

Fig. 9 Checking ram

\section{Command /uptime}

This command displays the length of time after the server is turned on. Like in Fig 10 below:

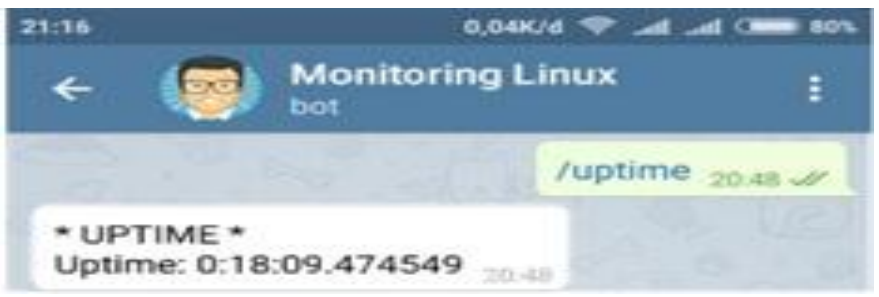

Fig. 10 Command /uptime

Checking uptime is also done manually, it aims to find out the notification goes according to the function that has been made or not. As in the following fig 11:

\section{telegramcubuntu:" $/$ tsms uptine

20:48:04 up 18 min, 1 user, load average: $0.00,0.04,0.11$

Fig. 11 Checking uptime

\section{Command /cpu}

This command displays the CPU used on the server. As in Fig 12 below:

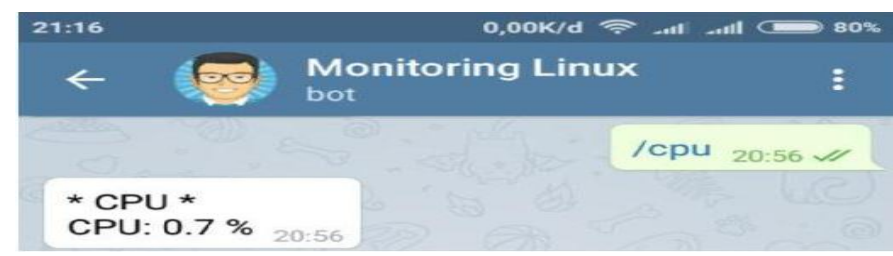

Fig. 12 Command /cpu

CPU checking is also done manually; it aims to find out the notification goes according to the function that has been made or not. As in the following fig 13: 
Ubuntu Server (1) [Berjalan] - Oracle VM VirtualBox

Berkas Mesin Tilik Masukan Peranti Bantuan

$\begin{array}{lr}\text { CPUI } 1 & 0.7 \%] \\ \text { Hen }[111111111 & 106 \mathrm{H} / .956] \\ \text { Sup [ } & 0 \mathrm{~K} / 3.726]\end{array}$

Fig. 13 Checking CPU

5. Command /disks

This command displays Disks on the server. As in Fig 14 below:

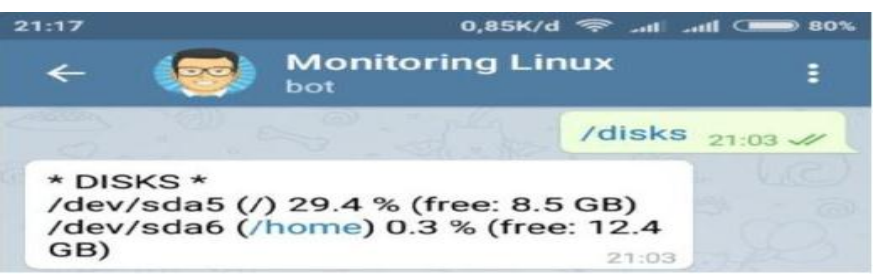

Fig. 14 Commands /disks

Checking Disks is also done manually, it aims to find out the notification goes according to the function that has been created or not. As in the following fig15:

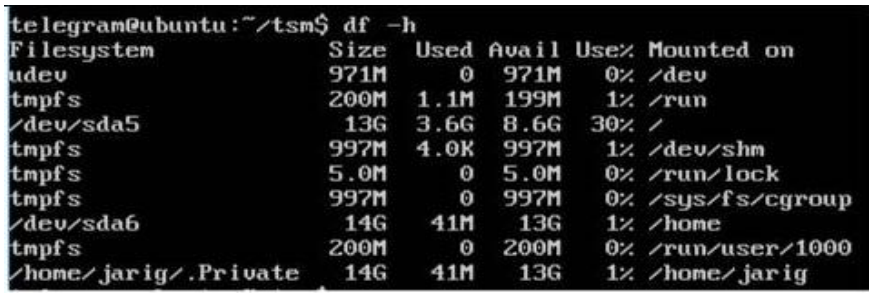

Fig. 15 Checking disk

\section{CONCLUSION}

In research conducted by the author, it can be concluded that by using a bot telegram can help the server administrator work in monitoring the server in real time. Can perform checks on the server, so that the admin who is not in place can find out the state of the server through the telegram application that has been installed on the smartphone. When the command processing time depends on an internet connection, the faster the internet connection is, the faster the bot executes or replies to the command.

\section{REFERENCES}

[1] M. Dunlop, S. Brewster, "The Challenge of Mobile Devices for HumanComputer Interaction". Pers. ubiquitous comput. 6.4, vol. 235-236. , 2002.

[2] T.M.T. Do, \& D. Gatica-Perez, "Human Interaction Discovery in Smartphone Proximity Networks", Person. and Ubiquitous Comput. 17.3, p: 413-431, 2011.

[3] J. Hill, et al., "Real conversations with artificial intelligence: a comparison between human-human online conversations and humanchatbot conversations", Comput. in Hum. Behav., vol. 49, pp. 245-250, 2015 .

[4] V. Hung, et al., "Towards a method for evaluating naturalness in conversational dialog systems", IEEE Int. Conf. of Syst., Man, and Cybern., San Antonio, pp. 1236-1241, 2009.

[5] M.B. Chaniago, A. Junaidi. "Student Presence Using Rfid And Telegram Messenger Application". 8th Widyatama Int. Seminar on Sustain. (WISS 2016), Widyatama University. and IEEE. Bandung. 2016.

[6] H. Setiaji and I.V. Paputungan, "Design of Telegram Bots for Campus Information Sharing," IOP Conf. Ser. Mater. Sci. Eng., vol. 325, no. 1, 2018.

[7] Y.B. Mulyana, "Linux Semudah Window”. Jakarta: PT Elex Media Komputindo, 2002.

[8] Schuerer, Katja, and C. Maufrais, Introduction to Programming using Python. Boston: Pr., pp. 1-242. 2010. 